Check for updates

Cite this: Mater. Chem. Front., 2021, 5, 7022

Received 21st May 2021 Accepted 25th July 2021

DOI: 10.1039/d1qm00755f

rsc.li/frontiers-materials

\title{
Self-organizing gelatin-polycaprplactone materials with good fluid transmission can promote full-thickness skin regeneration $\dagger$
}

\author{
Yifeng Nie, (D) $\ddagger^{a b}$ Xinxiao Han, $\ddagger^{a}$ Zhuo Ao, ${ }^{a b}$ Shangwei Ning, ${ }^{c}$ Xiang Li*ab and \\ Dong Han (D) ab
}

\begin{abstract}
Healing, reconstruction and recovery after skin injury have long been a major field of research, and many biomaterials have been designed for skin healing treatment. Because the skin repair process includes a series of complex processes such as hemostasis, inflammatory reactions and tissue remodeling, the microenvironment for bionic cell growth is particularly important. Herein, we used the self-organization effect of natural polymer materials to simulate the extracellular matrix, and prepared a structure-stable gelatin-polycaprplactone (Gt-PCL) through high-voltage electrospinning technology and a multiphase method. The composite material was capable of phase separation and showed a superior water retention rate and good fluid transmission effects in vivo and in vitro, which was used in repairing tests after full-thickness skin excision in rats. Gt-PCL materials showed not only good biocompatibility but also appropriate mechanical properties to effectively improve the epidermal healing rate and prevent wound expansion. RNA-sequencing and differential expression analysis showed that the polycaprolactone biomimetic formed a stable multi-stage, micro-nano composite structure with various soluble amino acids or proteins through self-organization. This material was able to repair the skin by up-regulating tissue repair-related genes, initiating neural repair and hair regeneration pathways and down-regulating the immune response and stress response-related pathways, thus avoiding secondary injury and excessive wound repair. Moreover, Itgax, a key mRNA associated with wound healing, was identified and has been shown to affect the immune and barrier profiles of the skin. Together, these results demonstrate that Gt-PCL materials promote full-thickness skin regeneration, and may have good clinical effects and potential application value in the future.
\end{abstract}

\section{Introduction}

Soft matter, an intermediate between solid and ideal fluid, is called a complex fluid. Its scale is between micro and macro, and it can spontaneously self-organize into mesoscopic physical structures. ${ }^{1}$ Large amounts of soft matter exist in organelles, cell membranes and entire cells in organisms. For example, tissues and cells, hierarchical structures formed by the selforganization of biological macromolecules, could be considered typical soft matter. ${ }^{2}$ The extracellular matrix (ECM), an

\footnotetext{
${ }^{a}$ CAS Center for Excellence in Nanoscience, National Center for Nanoscience and Technology, Beijing, 100190, P. R. China. E-mail: lixiang@nanoctr.cn

${ }^{b}$ School of Future Technology, University of Chinese Academy of Sciences, Beijing 100049, P. R. China

${ }^{c}$ College of Bioinformatics Science and Technology, Harbin Medical University,

Harbin 150081, P. R. China

$\dagger$ Electronic supplementary information (ESI) available. See DOI: 10.1039/ d1qm00755f

\$ These authors have contributed equally to this work.
}

important medium for material transport and energy exchange between cells, is also a typical soft matter structure in a fiber network system, which is synthesized and secreted by cells and formed by tissues. ${ }^{3,4}$ The extracellular matrix (ECM) is composed of two parts, the first part is interstitial gel, and the second part is fiber network. Interstitial gel is mainly composed of glycosaminoglycans and proteoglycan among them, so we use gelatin and collagen to simulate these substance. According to mechanical properties, biological activity and three-dimensional structure, and inspired by our previous research, ${ }^{5}$ we use the polycaprolactone (PCL) to simulate the structure of fiber network. These composite materials can provide biological and physical support for cell adhesion, proliferation, and differentiation, which are in line with the ideal tissue engineering structure, have good biocompatibility ${ }^{6-11}$ and mechanical requirements for tissue mechanical properties to use as an ideal tissue engineering dressing to repair tissues and skin. ${ }^{12,13}$ So we designed self-organized natural polymer materials to simulate the ECM, and prepared a structure-stable polycaprolactone (PCL) 
through high-voltage electrospinning technology and multiphase system separation and then this material was used in repairing tests after full-thickness skin excision in rats.

Skin injury is very common in animals and humans, and skin repair aims to restore skin function and achieve a scar-free appearance after wound repair. ${ }^{14,15}$ The soft matter fiber network and gel structure are the main components of skin tissue. ${ }^{16,17} \mathrm{We}$ prepared a structure-stable polymer PCL through high-voltage electrospinning and multiphase system separation, and the composite material was capable of phase separation and showed a superior water retention rate and good fluid transmission effects in vivo and in vitro. We examined the material in repairing tests after full-thickness skin excision in rats. To explore whether the designed micro-nano composite structure had good repair effects, we used two experimental groups, physiological saline (NEG group) and growth factor (POS group), to observe the effects after full-thickness skin resection in rats. We performed RNAsequencing (RNA-seq) and differential expression data analysis on skin materials to explore the skin repair mechanism at the molecular level. ${ }^{18,19}$ The results showed that the bionic structure (Gt-PCL) formed a stable multi-stage micro-nano composite with various soluble amino acids or proteins through self-organization. Gt-PCL repaired the skin by upregulating tissue repair-related genes, initiating neural repair and hair regeneration pathways and down-regulating the immune response and stress responserelated pathways, thus avoiding secondary injury and excessive wound repair. Moreover, Itgax, a key mRNA associated with Gt-PCL, which is known to influence the immune and barrier profiles of skin, was identified. ${ }^{20,21}$ Together, these results demonstrated that Gt-PCL promotes full-thickness skin regeneration, and may have good clinical effects and potential application value in the future.

\section{Results}

\subsection{Preparation of the self-organiztion Gt-PCL composite materials in vitro}

High-voltage electrospinning materials were prepared in a two-liquid phase system. With high-voltage electrospinning technology and the microscopic phase separation method, the Gt-PCL composite materials were composed of polycaprolactone (PCL, $\left.M_{\mathrm{n}}=70000-90000\right)$, gelatin and collagen, and the solvent was 2,2,2-trifluoroethanol $\left(\mathrm{C}_{2} \mathrm{H}_{3} \mathrm{~F}_{3} \mathrm{O}\right)$, which was used in repairing tests after full-thickness skin excision in rats. The whole overview of the study are shown in Fig. 1.

\subsection{Scanning/transmission electron microscopy and micromechanical elastic modulus tests}

After phase separation treatment, the stress of the selforganized composite Gt-PCL clearly changed. The quasi-static displacement scanning extension mode of a dynamic mechanical property analyzer was used for testing. The initial variable was $0.01 \%$, and the stretching speed was $20 \mu \mathrm{m} \mathrm{min}{ }^{-1}$. The sample size was $30 \mathrm{~mm} \times 2 \mathrm{~mm}$, and the sample clamping length was $10 \mathrm{~mm}$. The data obtained are shown in Fig. 2. After phase separation, greater applied stress was required to reach the same strain, and the elasticity and ductility of the material were better than those before phase separation. These features importantly indicate why the material is suitable as an experimental material for skin and dermal repair.

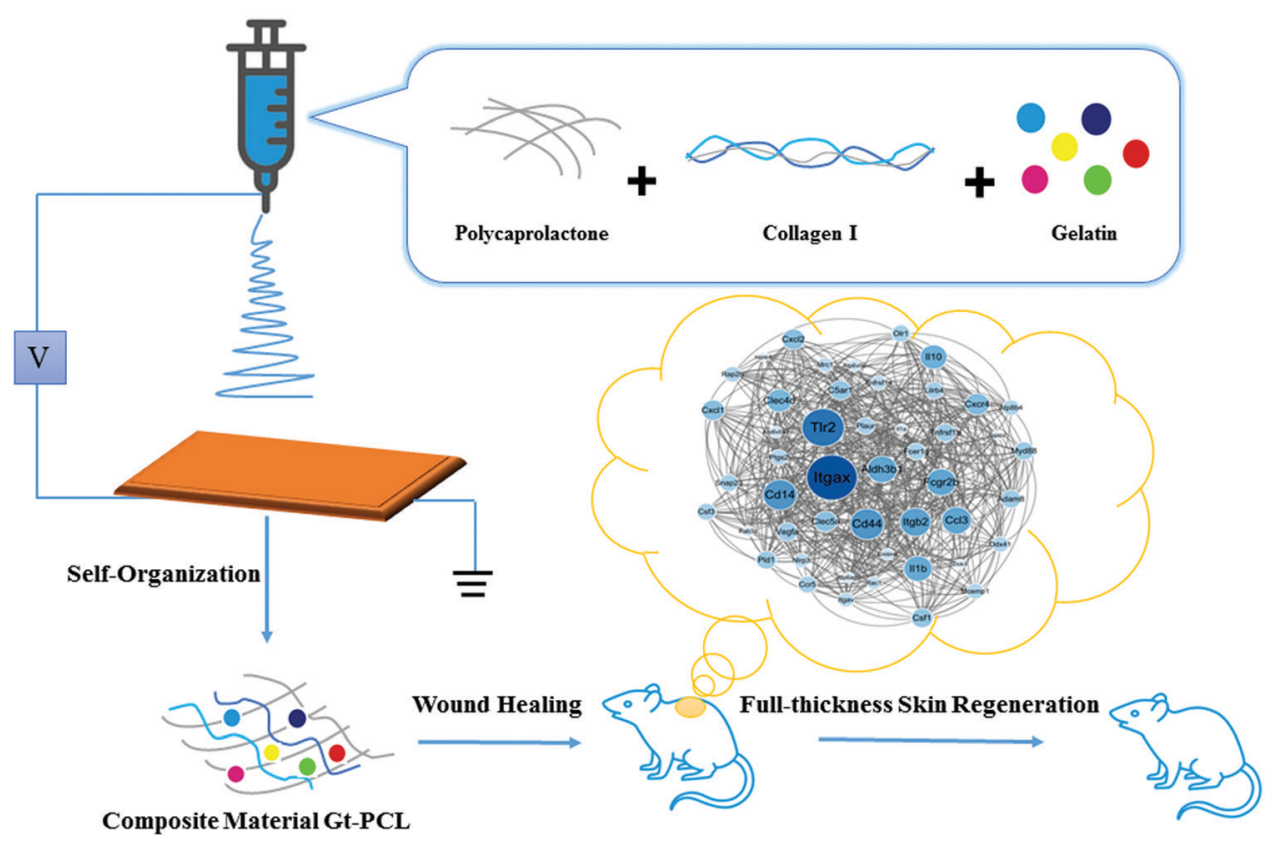

Fig. 1 A schematic overview of the study. Gt-PCL composite materials were synthesized with polycaprplactone, gelatin and collagen through high-voltage electrospinning technology and multiphase system separation, which was used in repairing tests after full-thickness skin excision in rats. Gt-PCL group showed an improved epidermal healing rate, promoted the proliferation and differentiation of fibroblasts, and was able to respond and repair in advance. Itgax as a key mRNAs associated with wound healing might serve as biomarkers of wound healing. 


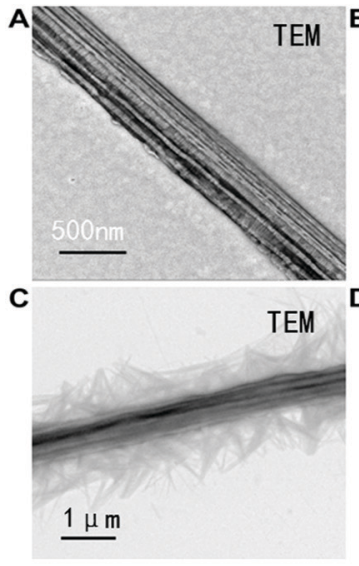

Time

$10 \mathrm{~min}$

$30 \mathrm{~min}$

$60 \mathrm{mim}$

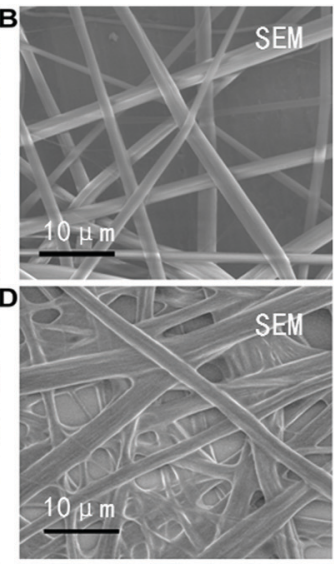

Before phase separation

$8 \mu \mathrm{m}$

$36 \mu \mathrm{m}$

$50 \mu \mathrm{m}$
$\mathbf{E}$

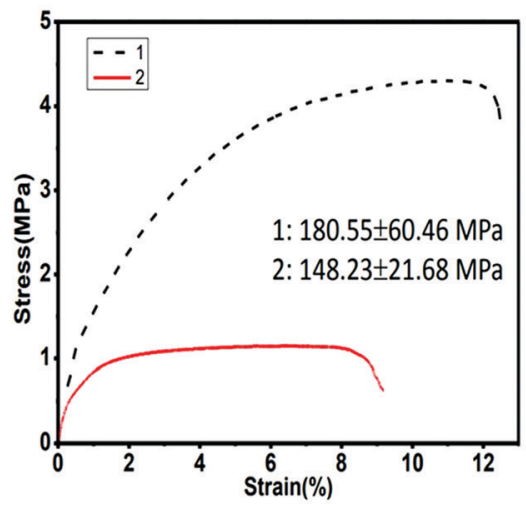

After phase separation

$3 \mu \mathrm{m}$

$12 \mu \mathrm{m}$

$16 \mu \mathrm{m}$

Fig. 2 Scanning/transmission electron microscope experiment and micromechanical elastic modulus test. (A) High resolution transmission electron microscope image: before material phase separation; (B) scanning electron microscope image: before material phase separation; (C) high resolution transmission electron microscope image: after material phase separation; (D) scanning electron microscope image: after material phase separation; (E) the stress-strain curves of self-organization composite materials before and after Gt-PCL phase separation. Red curve 1 and black curve 2 represent the material before and after phase separation treatment respectively.

\subsection{Self-organized composite Gt-PCL metabolism and water retention experiments}

The metabolic rate of Gt-PCL was calculated according to the following formula:

$$
R_{\mathrm{d}}=\frac{M_{0}-M_{30}}{M_{0}}
$$

where $R_{\mathrm{d}}$ is the metabolic rate, $M_{0}$ is the initial mass and $M_{30}$ is the mass on the 30th day. The final five groups of data were averaged. Finally, we determined that the self-organization composite Gt-PCL had an average metabolic rate of $66.27 \%$ within 30 days, whereas the average metabolic rate of single PCL in 30 days was only $13.01 \%$. The metabolic rate of Gt-PCL material was five times that of single PCL, thus indicating that Gt-PCL composite material have significantly better metabolic rate than that of single PCL in rats.

The water retention rate of Gt-PCL was calculated according to the following formula:

$$
R_{\mathrm{water}}=\frac{M_{\mathrm{wet}}-M_{\mathrm{dry}}}{M_{\mathrm{dry}}} .
$$

where $R_{\text {water }}$ is the water retention rate, $M_{\text {wet }}$ is the mass after water absorption and $M_{\text {dry }}$ is the mass before water absorption. The final five groups of data were averaged. We determined that the self-organized composite Gt-PCL had an average water retention rate of $390 \%$, whereas the average water retention rate of single PCL was only $72 \%$. The water content of animal skin is approximately $18-20 \%$ in the whole body. Therefore, the Gt-PCL materials ensures the proper water content during the dermal repair process to maintain the normal structure and function of skin.

\subsection{Fluid transmission experiment on Gt-PCL in vitro and in vivo}

The composite material Gt-PCL was completely soaked in distilled water and stirred overnight to form a liquid suspension, which was encapsulated with synthetic polyacrylamide hydrogel, as shown in Fig. 3A. The entire gel was soft, elastic and malleable (Fig. 3E), and the Gt-PCL white composite material encapsulated with polyacrylamide hydrogel clear visible, was evenly distributed and showed a good refractive index (Fig. 3B). First, in fluid transmission experiments in vitro, we dripped sodium fluorescein on one side at a speed of $25 \mu \mathrm{l} \mathrm{s}^{-1}$ with a micropipette (Fig. 3C). The solution quickly permeated the entire gel and reached the other side. We soaked and cleaned the composite gel three times with triple-distilled water and the entire gel still retained abundant yellow crystals (Fig. 3D), which eliminated fluid transmission channels on the gel surface, thereby indicating that the fluid transmission pathway was an internal network structure. We additionally conducted animal experiments on injured animal skin. Repeating the fluid transmission experiment in vitro, we applied composite gel to the skin wounds, and observed in the movement of fluorescein sodium at a speed of $25 \mu \mathrm{l} \mathrm{s}{ }^{-1}$ against gravity (Fig. 3F). The results were consistent with those observed in vitro (Fig. $3 \mathrm{H}$ ), and fluorescence electron microscopy showed that the gel was permeated with the complex with an interlaced green fluorescent network (Fig. 3I, bar: $50 \mu \mathrm{m}$ ). 


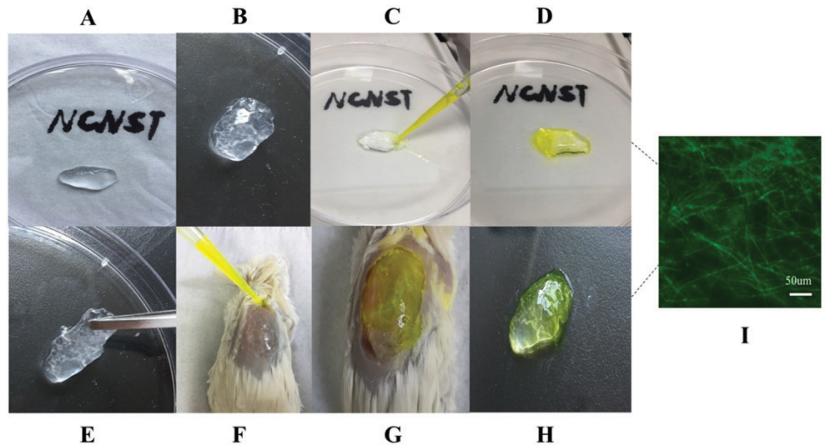

Fig. 3 Fluid transmission experiment of Gt-PCL materials in vitro and in vivo. (A and $\mathrm{B}$ ) The Gt-PCL composite material encapsulated with synthetic polyacrylamide hydrogel was evenly distributed and showed a good refractive index. (E) The entire gel was soft, elastic and malleable. ( $C$ and F) Sodium fluorescein were dripped on one side at a speed of $25 \mu \mathrm{l} \mathrm{s}^{-1}$ with a micropipette. ( $\mathrm{D}$ and $\mathrm{H}$ ) The solution quickly permeated the entire gel and reached the other side. (I) The gel was permeated with the complex with an interlaced green fluorescent network.

The above fluid transmission experiments demonstrated that the composite material Gt-PCL could transmit fluids efficiently and quickly in vivo and in vitro.

\subsection{Full-layer animal skin repair experiment}

The experimental results of Gt-PCL composite materials applied to full-layer skin repair are shown in Fig. 4A. Compared with the NEG group and POS group, the Gt-PCL composite materials group showed good performance in the wound healing process. Within 24 hours after modeling, the wound surfaces in rats showed hemostatic effects and dry surfaces. These observations differed from the general healing process and did not produce blood clots. Most importantly, owing to the viscosity of the material, the size of the wound shape did not increase with activity in rats, thus preventing secondary wound formation. We calculated the healing rate data for the composite Gt-PCL group, NEG group and POS group at different time points, as shown in Fig. 4B Compared with the control group, the composite Gt-PCL group showed improved epidermal healing rates and prevention of wound expansion in the first 7 days; thus, the composite has the ability to respond in advance.

We next performed hematoxylin-eosin (HE) staining in the full-layer skin repair experiment in rats (as shown in Fig. 4C). On the third day, the NEG group was still in the immune reaction period, and immune cells were still accounted for the majority, and the injured cells were constantly necrotic. In the POS group, many new capillaries were generated, fibroblastic cells and granulation tissue appeared, and relatively severe immune edema was observed in the surrounding normal tissues. Compared with two groups, the composite Gt-PCL group entered the final stage of immune response, an amount of new blood vessels and granulation tissue appeared, and many fibroblasts began to mature and differentiate. On the seventh day, both the POS group and the NEG group had unhealed epidermis at the edge of the wound and produced a certain pseudotumor-like epithelium. In the composite Gt-PCL group, with the supporting of the materials, the epidermis healed well, and the neonatal epidermis was uniform without pseudotumor-like epithelia. Moreover, many fibroblasts began

A
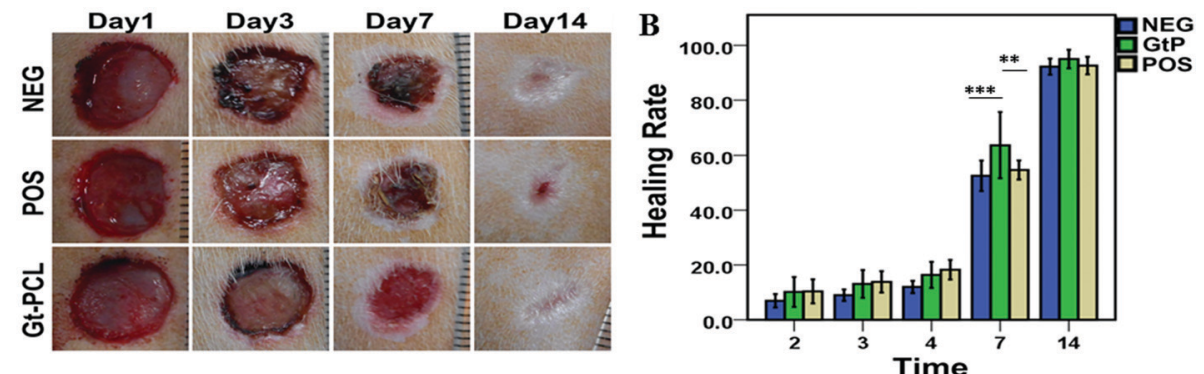

C

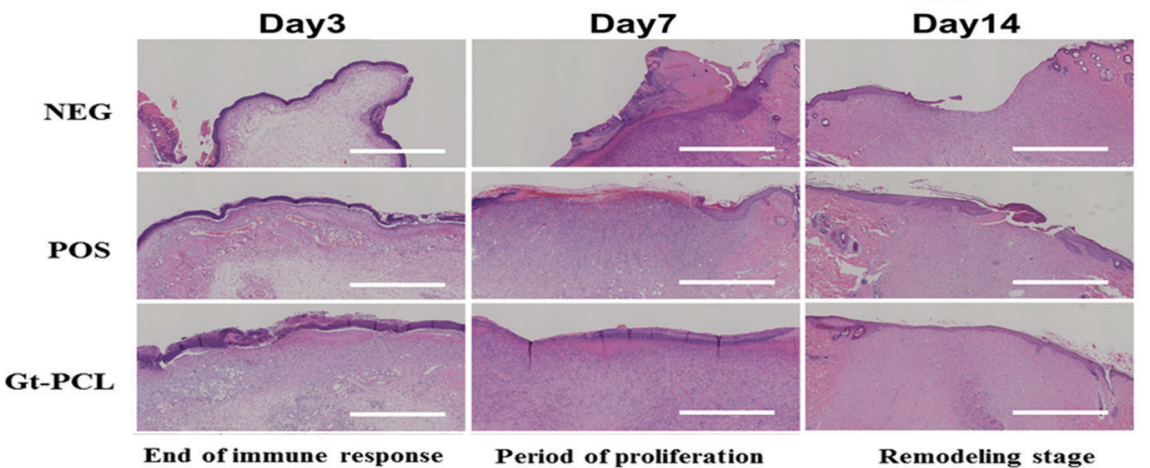

Fig. 4 Wound healing process in three groups of rats. (A) Photos of wound healing process. NEG: physiological saline group; POS: growth factor group; Gt-PCL: gelatin-polycaprplactone composite materials group. (B) The Figure is healing rate of full thickness skin repair process (** $p<0.01,{ }^{\star * *} p<0.001$ ). (C) Full-layer skin repair process samples fixed and stained with HE staining, Scale bar: $1 \mathrm{~mm}$. 
to mature into fibroblasts and enter the remodeling stage of the dermis. On the fourteen day, rats in the NEG group did not complete epidermal healing, and the neonatal epidermis was uneven, then the new collagen fibers produced by the neonatal fibroblasts were arranged in a disordered manner. The POS group showed excessive repair, many fibroblasts and new collagen fibers in a disordered arrangement. In the composite Gt-PCL group, the new epidermis was uniform and highly mature. Moreover, the stratification was better and did not show excessive repair. Most importantly, several hair follicles were repaired at edges of the wound, and collagen fibers of the newborn dermis were arranged in an orderly manner, and the structure was relatively uniform.

\subsection{Genome sequencing analysis of full-layer skin repair tissue}

The target genes were transcribed to RNA, and the sampling time point was at the end of inflammation reaction period and the beginning of proliferation period in the above three groups. At those times, many cytokines begin to be transcribed, and cells activated and proliferated, thus resulting in greater observable differential gene expression. ${ }^{22}$ After the sequencing data were processed through quality control of the original data and differential gene expression analysis, KEGG pathway enrichment analysis and GO gene function enrichment analysis were performed.

The KEGG pathway enrichment analysis results from transcriptome data indicated that in the Gt-PCL material group compared with the NEG group, as shown in Fig. 5A, the significantly up-regulated gene pathways included the Wnt signaling pathway, inflammation-mediated TRP pathway, Hippo signaling pathway, axon guidance pathway, Hedgehog signaling pathway, Rap1 signaling pathway and a signaling pathway that regulates stem cell pluripotency. Among them, the Wnt signaling pathway is also up-regulated in the normal repair process; it is a highly conserved signaling pathway, and it plays important roles in early embryo development, tissue regeneration and organ development. ${ }^{23,24}$ The signaling pathway regulating pluripotency of stem cells regulates pluripotent stem cells in wound repair. The Hippo signaling pathway is a signaling pathway with Hippo protein as the core protein, whose up-regulation promotes tissue proliferation. ${ }^{25-27}$ Axon guidance (also known as axon pathfinding) is an important pathway that controls the direction of axon growth during nerve development and plays an important role in axon position. ${ }^{28,29}$ Hedgehog, the core gene of the Hedgehog signaling pathway, is a segmented polar gene mainly involved in cell differentiation and maintaining cell dryness during development. ${ }^{30-32}$ Rap1 protein in the Rap1 signaling pathway is also an important regulatory protein in the NF- $\mathrm{KB}$ signaling pathway, ${ }^{33}$ which has prominent antibacterial effects. ${ }^{34}$

The GO results of in the material group and NEG group showed significant up-regulation (as shown in Fig. 5B) mainly in skin development, tissue development, epithelium development, hair cycle, tissue morphogenesis and epithelial cell differentiation pathways. The above sequencing results on skin full-thickness repair tissue demonstrated that the composite Gt-PCL improved the healing rate of epidermis and was able to promote full-thickness skin regeneration through multiple signaling pathways.

RNA-seq was performed for the NEG, POS and material groups to characterize the molecular features of full-layer skin repair. Most differentially expressed mRNAs and long noncoding RNAs(lncRNAs) were specific in diverse conditions (Fig. 6A and B). Among the groups, Gt-PCL showed more differences in mRNAs and IncRNAs (Fig. 6C), and the
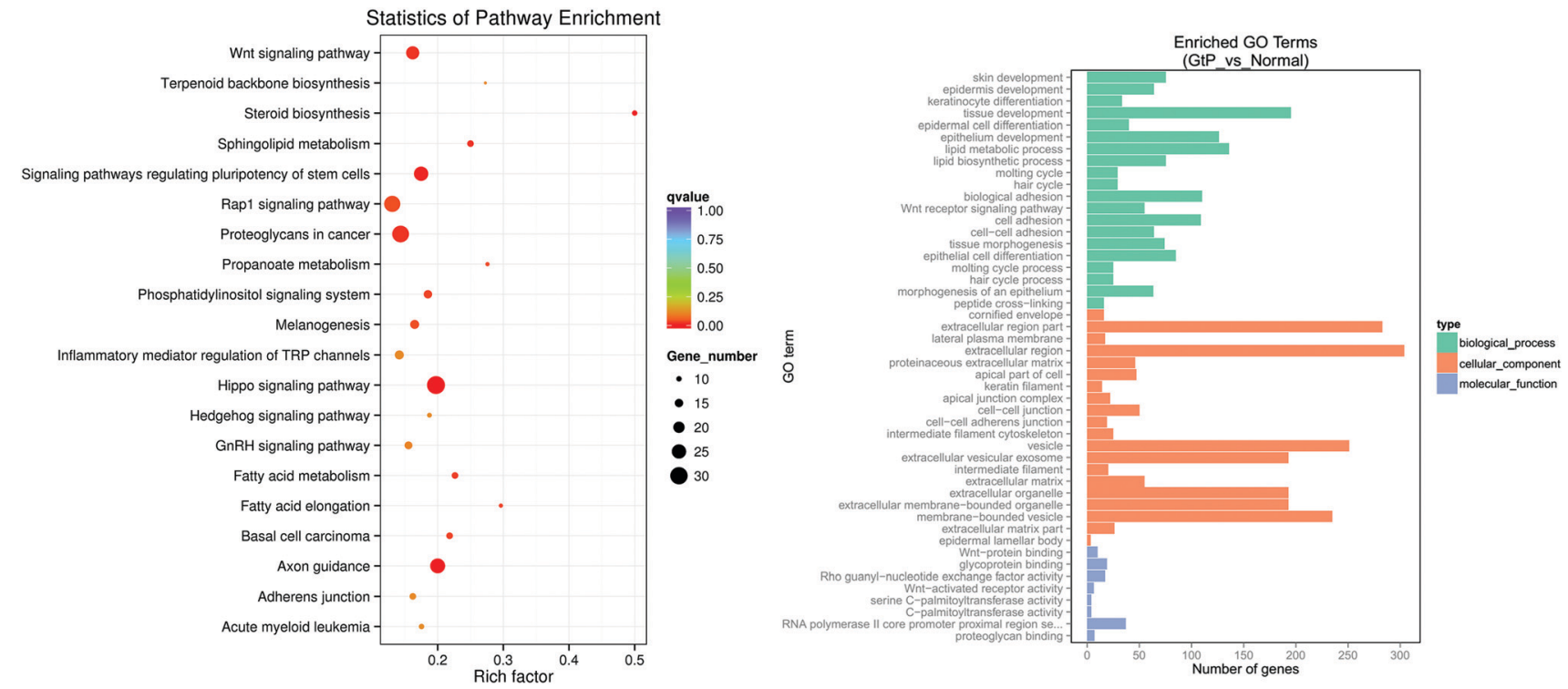

Fig. 5 KEGG Pathway and GO terms of up-regulated of genes, Gt-PCL vs. NEG. The results showed that the GO gene functions were concentrated in the material group and the NEG group, in which green represented the genes involved in biological processes, red represented the genes involved in cell component synthesis, and blue represented the genes involved in functional adjustment. 

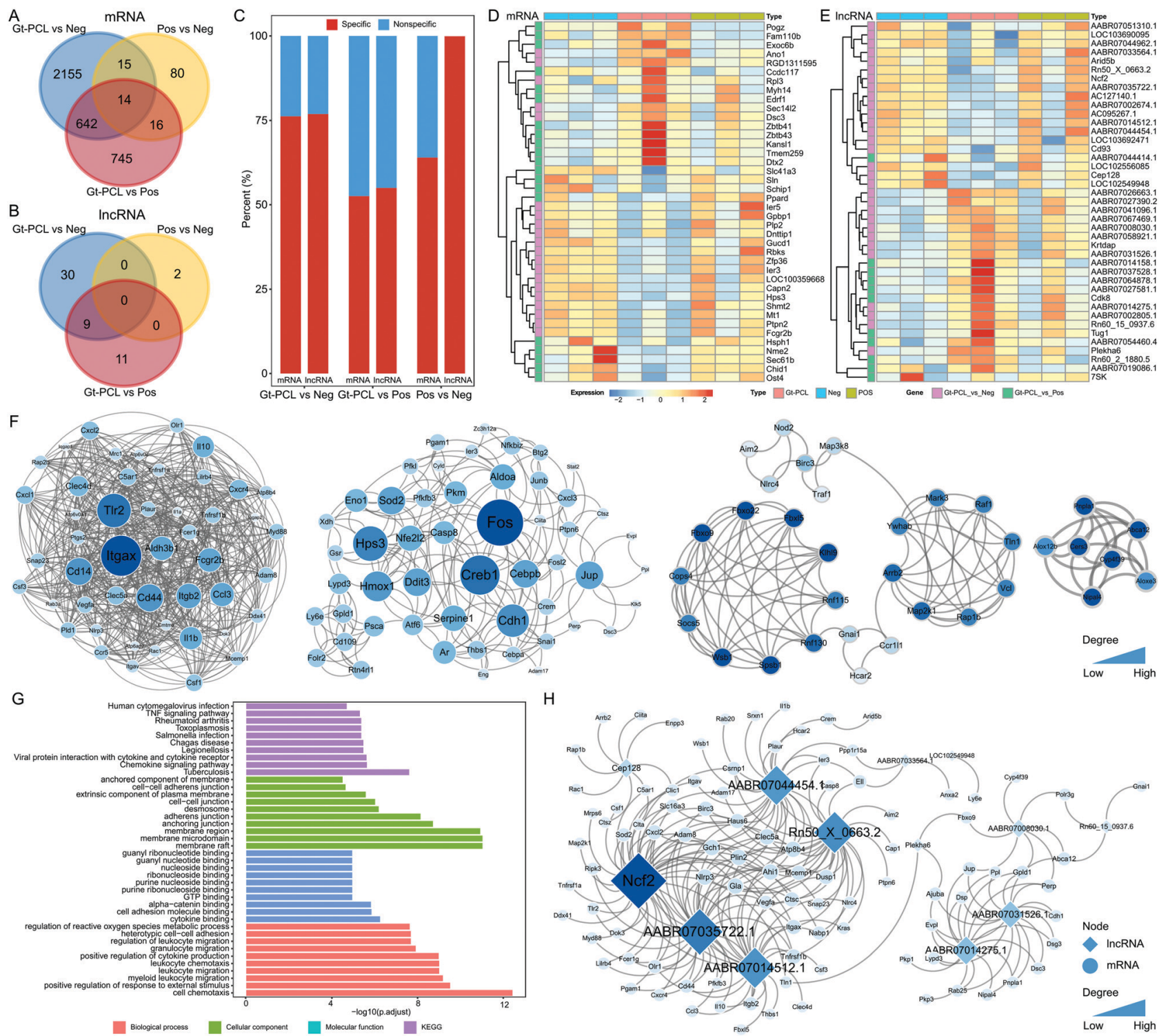

Fig. 6 Analysis and interaction network of three groups of differentially expressed mRNAs and IncRNAs. (A and B) Venn diagrams of mRNAs and lncRNAs in NEG, POS and material groups. (C) Bar charts of mRNAs and IncRNAs. (D and E) Cluster analysis of mRNAs and IncRNAs in NEG, POS and material groups. (F) Four core sub-networks were identified and analyzed for differentially expressed mRNAs. (G) Functional analysis of core sub-networks. (H) Co-expression of IncRNAs.

differentially expressed mRNAs and lncRNAs clustered according to the NEG, POS and material groups (Fig. 6D and E). The result indicated that differentially expressed mRNAs and lncRNAs might serve as biomarkers of wound healing. We also constructed two global interacted networks for differential mRNAs to explore the relationships of specific genes in the Gt-PCL group (Fig. S5A and B, ESI $\dagger$ ). Four core sub-networks were identified and analyzed for differentially expressed mRNAs in Gt-PCL vs. the NEG group (Fig. 6F). Some key mRNAs associated with Gt-PCL were found. For example, Itgax, a key mRNA in the first core sub-network, has been demonstrated to be associated with immune and barrier profiles of the skin. ${ }^{20,21}$ In addition, functional analysis of core sub-networks showed that differentially expressed mRNAs were associated with the TNF signaling pathway, cell-cell adhesion, skeletal muscle tissue development and other full-thickness skin repair functions (Fig. 6G and Fig. S5C, ESI $\dagger$ ). The differentially expressed mRNAs and lncRNAs also showed close interactions, on the basis of co-expression (Fig. 6G and Fig. S5D, ESI $\dagger$ ). Moreover, some key functional lncRNAs were also identified through this network. For example, TUG1 was a key IncRNA in the mRNA-IncRNA interaction network for the Gt-PCL group $v s$. the POS group (Fig. S5D, ESI $\dagger$ ). Previous studies have demonstrated that the IncRNA TUG1 promotes cell growth and angiogenesis, thus aiding in wound repair. ${ }^{35,36}$ Together, these results indicate that Gt-PCL leads to particular changes at the molecular level, and the observed changes in the expression of mRNAs and IncRNAs also explain the biological mechanism underlying Gt-PCL's favorable curative effects. 


\section{Discussion}

A structure-stable polymer Gt-PCL, was synthesized with polycaprplactone, gelatin and collagen through high-voltage electrospinning technology and multiphase system separation. We used the self-organization effect of natural polymer material to simulate ECM. The composite material was capable of phase separation, showed an excellent metabolic rate and superior water retention rate and had good fluid transmission effects in vivo and in vitro, which was used in repairing tests after fullthickness skin excision in rats. Compared with the control group, the composite Gt-PCL group showed improved epidermal healing rates, prevented wound expansion in the first 7 days, and was able to respond in advance. RNA-seq and differential expression analysis showed that the composite Gt-PCL formed a stable multi-stage micro-nano composite structure with various soluble amino acids or proteins through self-organization. KEGG pathway enrichment and GO gene function clustering analysis of the material group and NEG group indicated that the material group provided effective antibacterial and wound environmental protection. Skin repair occurred through up-regulation of tissue repair-related genes, initiation of neural repair pathways and hair regeneration pathways, and down-regulation of immune response and stress response-related pathways, thus avoiding secondary injury and excessive wound repair. Therefore, the material group showed good antibiosis, protection and restoration of the local microenvironment in early stages of the wound immune response and the beginning stage of remodeling.

The composite Gt-PCL material has favorable biological effects in the wound repair process, as mainly reflected in the immune response and cell stress response. The normal immune response is beneficial for tissue repair, potentially causing secondary injury or excessive shrinkage. The Gt-PCL material group showed activation of many antibacterial and immune-related gene pathways, thus providing protection and support for the wound. For example, the Rap1 protein in the Rap1 signaling pathway is an important regulatory protein in the NF- $\kappa \mathrm{B}$ signaling pathway, which is associated with antibacterial effects. Second, the Gt-PCL material group showed activation of many functional gene pathways associated with morphogenesis, stem cell generation and embryonic development. For example, the Wnt signaling pathway, a signaling pathway regulating stem cell pluripotency, could promote early embryonic development, tissue regeneration and organ development and the Hippo signaling pathway promote tissue proliferation. The core gene Hedgehog in the Hedgehog signaling pathway is a segmented polar gene, which is mainly involved in cell differentiation during development and in maintaining cell proliferation. Most importantly, the Gt-PCL material group initiated a process of nerve regeneration, which is associated with hair regeneration, during this period. Axon guidance (also known as axon pathfinding), an important pathway controlling the growth direction of axons in neurodevelopment, plays an important role in axon positioning. At present, nervous system regeneration and hair functional recovery are also major areas of research in skin tissue regeneration.

These differentially expressed mRNAs and IncRNAs clustered in three groups corresponding to the NEG, POS and Gt-PCL groups. The differentially expressed mRNAs and lncRNAs might serve as biomarkers of wound healing. We also constructed two global interacted networks for differential mRNAs to explore the relationships among specific genes in the Gt-PCL group. Itgax, identified as a key mRNA in the first core sub-network, has been demonstrated to be associated with immune and barrier profiles of skin. In addition, the functional analysis of core sub-networks showed that differentially expressed mRNAs were associated with the TNF signaling pathway, cell-cell adhesion, skeletal muscle tissue development and other full-thickness skin repair functions. Moreover, some key functional lncRNAs were identified. For example, TUG1 was found to be a key lncRNA in the mRNA-lncRNA interaction network for the Gt-PCL vs. POS group. Previous studies have demonstrated that the IncRNA TUG1 promotes cell growth and angiogenesis, thus facilitating wound repair. Together, these results indicate that Gt-PCL leads to particular changes at the molecular level, and the changes in the expression of mRNAs and lncRNAs explain the biological mechanism underlying Gt-PCL's favorable curative effects.

\section{Conclusion}

In summary, with high-voltage electrospinning technology and the microscopic phase separation method, we successfully used polycaprplactone, gelatin and collagen to synthesize the Gt-PCL composite materials, which self-organizes and simulates ECM. This composite material was capable of phase separation, and showed a superior water retention rate and good fluid transmission effects in vitro and in vivo. Gt-PCL was used in repairing tests after full-thickness skin excision in rats. Compared with the control group, the composite Gt-PCL group showed an improved epidermal healing rate, promoted the proliferation and differentiation of fibroblasts, and was able to respond and repair in advance. RNA-seq and differential expression data analysis showed that the Gt-PCL formed a stable multi-stage micro-nano composite structure with various soluble amino acids or proteins through self-organization. The material was able to repair the skin by up-regulating tissue repair-related genes, initiating neural repair pathways and hair regeneration pathways and down-regulating the immune response and stress response-related pathways, thereby avoiding secondary injury and excessive wound repair, and improving the epidermal healing rate. Moreover, Itgax, a key mRNAs associated with wound healing, was identified and has been demonstrated to associate with immune. In addition, TUG1, a key lncRNA, has been demonstrated to promote cell growth and angiogenesis, thus aiding in repair and preventing wound expansion. These RNAs might serve as biomarkers of wound healing. Therefore, our results demonstrate that Gt-PCL composite materials effectively promotes full-thickness skin 
regeneration, and may have good clinical effects and potential application value in the future.

\section{Experimental section}

\subsection{Preparation of PCL through high-voltage electrospinning} technology

High-purity PCL with a molecular weight of 70 000-90 000 was dissolved in a mixed solution of dichloromethane and $N$, $N$-dimethylformamide (in a ratio of $1: 4, \mathrm{v} / \mathrm{v}$ ), ${ }^{37,38}$ and fully stirred on a magnetic stirrer for 6-8 hours, so that the PCL particles were fully dissolved in a transparent liquid. The electrospinning liquid was added with a micro injection pump in high-pressure electrospinning, and the collected material was placed in a vacuum drying oven for more than 5 hours to ensure complete evaporation of all volatile solvents.

\subsection{Fiber phase separation treatment}

Before the material was used, $N$-hydroxysuccinimide and 1-(3dimethylaminopropyl)-3-ethylcarbodiimide hydrochloride (NHS/ EDC) mixed solution $\left(0.1 \mathrm{~mol} \mathrm{l}^{-1}\right)$ was prepared with MES buffer solution, and the above-mentioned high-voltage electrostatic spinning materials were cut to an appropriate size, soaked in NHS/ EDC solution for 20 minutes, and then soaked and cleaned with physiological saline before use.

\subsection{Preparation of the self-organized composite material Gt-PCL}

High-voltage electrospinning materials were prepared in a two-liquid phase system. The materials used were PCL $\left(M_{\mathrm{n}}=70000-90000\right)$, gelatin and collagen, and the solvent was trifluoroethanol (TFE, $\left.\mathrm{C}_{2} \mathrm{H}_{3} \mathrm{~F}_{3} \mathrm{O}\right)$. In a mass ratio of $2: 1: 1(\mathrm{w} / \mathrm{w} / \mathrm{w}), 2 \mathrm{~g}$ PCL, gelatin and collagen were added to each $10 \mathrm{ml}$ of TFE and continuously stirred for 4 hours with a magnetic stirrer. The heating temperature was $40{ }^{\circ} \mathrm{C}$ to enable the solute to fully dissolve into a uniform liquid, and the electrospinning liquid was added with a micro-injection pump for high-voltage electrospinning. ${ }^{39,40}$ Finally, the collected composite electrospinning materials were vacuum dried overnight.

\subsection{Scanning electron microscopy and transmission electron microscopy}

The materials before and after phase separation were prepared, soaked in $2.5 \%$ glutaraldehyde, fixed overnight at $4{ }^{\circ} \mathrm{C}$ and then dehydrated by gradient. Finally, the samples were dried with the critical point drying method and scanned with a scanning electron microscope in low vacuum mode. In accordance with the above conditions for high-voltage electrospinning, PCL was prepared directly on copper mesh, and the materials before and after phase separation were prepared and imaged with a transmission electron microscope (Hitachi, H-7500, Japan).

\subsection{Micromechanical elastic modulus tests}

The quasi-static displacement scanning extension mode of a dynamic mechanical property analyzer was used for testing. The initial variable was $0.01 \%$, and the stretching speed was
$20 \mu \mathrm{m} \mathrm{min}{ }^{-1}$. The sample size was $30 \mathrm{~mm} \times 2 \mathrm{~mm}$, and the sample clamping length was $10 \mathrm{~mm}$.

\subsection{Metabolism experiment of self-organized composite Gt-PCL}

Standard enzyme solution (collagen I, concentration $0.02 \mathrm{U} \mathrm{ml}^{-1}$ ) was prepared by DPBS. Five pieces of $2 \mathrm{~cm} \times 2 \mathrm{~cm}$ composite Gt-PCL and a single PCL of similar thickness were selected for experimental comparison. Each sample was weighed and tested separately. After weighing, each sample was soaked in $0.02 \mathrm{U} \mathrm{ml}^{-1}$ standard enzyme solution and placed in a cell incubator for 30 days at $37{ }^{\circ} \mathrm{C}$, and new standard enzyme solution was replaced on the 15th day. After 30 days, all samples were washed with phosphate buffer three times and then freeze-dried. After freezedrying, each sample was weighed, and the data were statistically processed in Origin Pro 9.1.

\subsection{Water retention experiment of self-organized composite Gt-PCL}

Five pieces of $2 \mathrm{~cm} \times 2 \mathrm{~cm}$ composite Gt-PCL and a single PCL with similar thickness were selected for experimental comparison. All samples were weighed, then soaked in phosphate buffer for 20 minutes, blotted with absorbent paper to remove excess water and weighed again. The data were statistically processed in Origin Pro 9.1.

\subsection{Fluid transmission experiment on Gt-PCL in vitro and in vivo}

We prepared $10 \mathrm{ml}$ of mixed solution containing $2.5 \mathrm{ml}$ acrylamide solution $(40 \%, \mathrm{w} / \mathrm{v}), 0.5 \mathrm{ml}$ bis-acrylamide solution $(2 \%, \mathrm{w} / \mathrm{v})$ and $7 \mathrm{ml}$ pure water, which was fully shaken and uniformly mixed on a vortex oscillator. Subsequently, 1\% (total volume of use) of ammonium persulfate solution $(10 \% \mathrm{w} / \mathrm{v})$ and $1 \%$ of tetramethylethylenediamine were added, fully shaken and mixed uniformly, and the composite material Gt-PCL was completely soaked in distilled water and stirred overnight to form a liquid suspension. Synthetic polyacrylamide hydrogel was used to wrap and sodium fluorescein was dripped on one side to observe the fluid transmission to the other side. Injured animal skin was used as the substrate; the appropriate composite material was applied to skin wounds, and the fluid transmission experiment in vitro was repeated.

\subsection{Full-thickness skin repair experiment}

After 20 SPF Wistar male rats 6 weeks of age and weighing approximately 300-350 g were completely anesthetized, the back skin was depilated with a depilator, the experimental site was wiped with $75 \%$ ethanol and air dried and three holes with a diameter of $1 \mathrm{~cm}$ were created at $1.5 \mathrm{~cm}$ from up each of the left and right sides of the spinal column and labeled No. 1 to No. 6. The six wounds on each rat were treated with the following in randomized order: physiological saline (NEG group), growth factor (POS group) and micro-nano bionic structure (material group). The skin in the selected area was excised and deeply cut to the superficial fascia of subcutaneous muscle with sterile surgical instruments. After the model was 
established, every wound in each rat was photographed at a fixed distance every 24 hours for 14 days, and the healing rate was calculated in OriginPro 9.1. All the animal experiments were complied with relevant laws and were approved by the Ethics Committee of National Centre for Nanoscience and Technology (NCNST).

\subsection{Expression procedure in diverse groups of rats and network analysis of LncRNA and mRNA mapping}

Libraries were sequenced with the Illumina HiSeq 4000 platform with a 125-bp pair-end sequencing strategy. Raw data (raw reads) in fastq format were first processed. In this step, clean data (clean reads) were obtained by removal of reads containing adapters, reads containing poly-N and low quality reads from the raw data. ${ }^{41}$ Q20, Q30 and the GC content of the clean data were calculated. ${ }^{42,43}$ Reference genome and gene model annotation files were directly downloaded (http://genome.ucsc.edu). Read mapping was performed with HISAT2, StringTie and HTseq. The expected number of fragments per kilobase of transcript sequence per million base pairs sequenced was calculated with StringTie-eB. ${ }^{44,45}$

\subsection{Statistical analysis}

Statistical analysis was performed in SPSS 21.0 software. Student's $t$-test was used for comparison between two groups, and the $P$ values were corrected with the FDR. $P<0.05$ was considered statistically significant.

\section{Author contributions}

Yifeng Nie wrote the main manuscript text, and together with Dong Han designed experiments. Yifeng Nie and Xinxiao Han performed the experiments and Xiang Li supervised the project. Zhuo Ao and Shangwei Ning supported the process of analyzing results. All authors discussed the results and reviewed the manuscript.

\section{Conflicts of interest}

There are no conflicts to declare.

\section{Acknowledgements}

This work was supported by Key Research Program of Frontier Sciences of CAS (No.ZDBS-LY-SLH036).

\section{References}

1 X. W. Du, J. Zhou, J. F. Shi and B. Xu, Supramolecular hydrogelators and hydrogels: From soft matterto molecular biomaterials, Chem. Rev., 2015, 115, 13165-13307.

2 A. T. Waigh, Advances in the microrheology of complex fluids, Rep. Prog. Phys. Phys. Soc., 2016, 79, 74601.
3 M. F. Brizzi, G. Tarone and P. Tarone, Extracellular matrix, integrins, and growth factors as tailors of the stem cell niche, Curr. Opin. Cell Biol., 2012, 24, 645-651.

4 I. Levental, P. Georgesa and P. Janmey, Soft biological materials and their impact on cell function, Soft Matter, 2006, 2, 1-9.

5 J. T. Feng, W. Fang, X. X. Han, Z. Ao, Q. M. Sun and D. Han, A "green pathway" different from simple diffusion in soft matter: Fast molecular transport within micro/nanoscale multiphase porous systems, Nano Res., 2014, 7, 434-442.

6 H. Yoshimoto, Y. Shin and H. Terai, et al., A biodegradable nanofiber scaffold by electrospinning and its potential for bone tissue engineering, Biomaterials, 2003, 24(12), 2077-2082.

7 S. H. Oh, I. K. Park and J. M. Kim, et al., In vitro and in vivo characteristics of PCL scaffolds with pore size gradient fabricated by a centrifugation method, Biomaterials, 2007, 28(9), 1664-1671.

8 Y. Tabata and Y. Ikada, Protein release from gelatin matrices, Adv. Drug Delivery Rev., 1998, 31(3), 287-301.

9 M. C. Gómez and M. E. Caballero, et al., Functional and bioactive properties of collagen and gelatin from alternative sources: A review, Food Hydrocolloids, 2011, 25(8), 1813-1827.

10 Z. Chen, X. Mo and F. Qing, Electrospinning of collagenchitosan complex, Mater. Lett., 2007, 61(16), 3490-3494.

11 S. A. Sell, M. J. Mcclure and K. Garg, et al., Electrospinning of collagen/biopolymers for regenerative medicine and cardiovascular tissue engineering, Adv. Drug Delivery Rev., 2009, 61(12), 1007-1019.

12 R. O. Hynes, The extracellular matrix: Not just pretty fibrils, Science, 2009, 326, 1216-1219.

13 C. Bonnans, J. Chou and Z. Werb, Remodelling the extracellular matrix in development and disease, Nat. Rev. Mol. Cell Biol., 2014, 15, 786-801.

14 B. S. Kim, Y. W. Kwon and J. S. Kong, 3D cell printing of in vitro stabilized skin model and in vivo pre-vascularized skin patch using tissue-specific extracellular matrixbioink: A step towards advanced skin tissue engineering, Biomaterials, 2018, 168, 38-53.

15 S. Ahn, C. O. Chantre and A. R. Gannon, Soy protein/ cellulose nanofiber scaffolds mimicking skin extracellular matrix for enhanced wound healing, Adv. Healthcare Mater., 2018, 7, 170-175.

16 L. W. Xia, R. Xie, X. J. Ju, W. Wang, Q. M. Chen and L. Y. Chu, Nano-structured smart hydrogels with rapid response and high elasticity, Nat. Commun., 2013, 4, 2226-2237.

17 J. Kopecek, Hydrogel biomaterials: A smart future, Biomaterials, 2007, 28, 5185-5192.

18 F. Ozsolak and P. M. Milos, RNA sequencing: Advances, challenges and opportunities, Nat. Rev. Genet., 2011, 12, 87-98.

19 S. A. Byron, K. R. Van, D. M. Engelthaler, J. D. Carpten and D. W. Craig, Translating RNA sequencing into clinical diagnostics: Opportunities and challenges, Nat. Rev. Genet., 2016, 17, 257-271. 
20 G. Hom, R. R. Graham and B. Modrek, Association of systemic lupus erythematosus with C8orf13-BLK and ITGAM-ITGAX, N. Engl. J. Med., 2008, 358, 900-909.

21 S. H. Han, K. H. Xana and D. Harshal, Evaluation of imputation-based association in and around the integrinalpha-M (ITGAM) gene and replication of robust association between a non-synonymous functional variant within ITGAM and systemic lupus erythematosus (SLE), Hum. Mol. Genet., 2009, 18, 1171-1180.

22 C. Trapnell, A. Roberts, L. Goff, G. Pertea, D. Kim and D. R. Kelley, Differential gene and transcript expression analysis of RNA-seq experiments with TopHat and Cufflinks, Nat. Protoc., 2012, 7, 562-578.

23 C. Y. Logan and R. Nusse, The Wnt signaling pathway in development and disease, Annu. Rev. Cell Dev. Biol., 2004, 20, 781-810.

24 T. P. Rao and K. Michael, An updated overview on Wnt signaling pathways: a prelude for more, Circ. Res., 2010, 106, 1798-1806.

25 X. Varelas and B. W. Miller, The Hippo pathway regulates Wnt/beta-catenin signaling, Dev. Cell, 2010, 18, 579-591.

26 J. Huang, S. Wu, J. Barrera, K. Matthews and D. J. Pan, The Hippo signaling pathway coordinately regulates cell proliferation and apoptosis by inactivating Yorkie, the Drosophila Homolog of YAP, Cell, 2005, 122, 421-434.

27 A. Ramos and F. D. Camargo, The Hippo signaling pathway and stem cell biology, Trends Cell Biol., 2012, 22, 339-346.

28 D. J. Barry, Molecular mechanisms of axon guidance, Science, 2002, 298, 1959-1964.

29 Z. L. Hua, H. Chang, Y. S. Wang, P. M. Smallwood and J. Nathans, Partial interchangeability of Fz3 and Fz6 in tissue polarity signaling for epithelial orientation and axon growth and guidance, Development, 2014, 141, 3944-3954.

30 P. W. Ingham and A. P. McMahon, Hedgehog signaling in animal development: Paradigms and principles, Genes Dev., 2010, 15, 3059-3087.

31 P. Mill, R. Mo and $\mathrm{H}$. Fu, Sonic hedgehog-dependent activation of Gli2 is essential for embryonic hair follicle development, Genes Dev., 2003, 17, 282-294.

32 I. Brownell, E. Guevara, C. B. Bai, C. A. Loomis and A. L. Joyner, Nerve-derived sonic hedgehog defines a niche for hair follicle stem cells capable of becoming epidermal stem cells, Cell Stem Cell, 2011, 8, 552-565.

33 D. S. Regier, J. Higbee, K. M. Lund, F. Sakane, S. M. Prescott and M. K. Topham, Diacylglycerol kinase iota regulates Ras guanyl-releasing protein 3 and inhibits Rap1 signaling, Proc. Natl. Acad. Sci. U. S. A., 2005, 102, 595-600.
34 F. G. Osorio and V. C. Soria, NF-кB signaling as a driver of ageing, Int. Rev. Cell Mol. Biol., 2016, 326, 133-174.

35 M. D. Huang, W. M. Chen, F. Z. Qi, M. Sun, T. P. Xu, P. Ma and Y. Q. Shu, Long non-coding RNA TUG1 is up-regulated in hepatocellular carcinoma and promotes cell growth and apoptosis by epigenetically silencing of KLF2, Mol. Cancer, 2015, 14, 165.

36 R. Dong, G. B. Liu, G. Chen, K. Li, S. Zheng and K. R. Dong, Targeting long non-coding RNA-TUG1 inhibits tumor growth and angiogenesis in hepatoblastoma, Cell Death Dis., 2016, 7, 227.

37 M. Labet and W. Thielemans, Synthesis of polycaprolactone: A review, Chem. Soc. Rev., 2009, 38, 3484-3504.

38 J. M. Williams, A. Adewunmi, R. M. Schek, C. L. Flanagan, P. H. Krebsbach, S. E. Feinberg, S. J. Hollister and S. Das, Bone tissue engineering using polycaprolactone scaffolds fabricated via selective laser sintering, Biomaterials, 2005, 26, 4817-4827.

39 Y. Keereeta, T. Thongtem and S. Thongtem, Synthesis of lanthanum tungstate interconnecting nanoparticles by high voltage electro spinning, Appl. Surf. Sci., 2015, 351, 1075-1080.

40 N. Arun, V. Aravindan, S. Jayaraman, N. Shubha, W. C. Ling, S. Ramakrishna and S. Madhavi, Exceptional performance of a high voltage spinel $\mathrm{LiNi}_{0.5} \mathrm{Mn}_{1.5} \mathrm{O}_{4}$ cathode in all one dimensional architectures with an anatase $\mathrm{TiO}_{2}$ anode by electrospinning, Nanoscale, 2014, 6, 8926-8934.

41 Y. F. Nie, Y. He, D. Han, X. Li and Y. S. Liu, Amiodarone inhibits arrhythmias in hypertensive rats by improving myocardial biomechanical properties, Sci. Rep., 2020, 10, 21565.

42 Q. W. Wang, B. Chen, M. Cao, J. F. Sun, H. Wu, P. Zhao, J. Xing, Y. Yang, X. Q. Zhang, M. Ji and N. Gu, Response of MAPK pathway to iron oxide nanoparticles in vitro treatment promotes osteogenic differentiation of hBMSCs, Biomaterials, 2016, 86, 11-20.

43 J. L. Rinn and H. Y. Chang, Genome regulation by long noncoding RNAs, Annu. Rev. Biochem., 2012, 81, 145-166.

44 R. A. Gupta, N. Shah, K. C. Wang, J. Kim, H. M. Horlings, D. J. Wong, M. C. Tsai, T. Hung, P. Argani, J. L. Rinn, Y. L. Wang and P. Brzoska, Long non-coding RNA HOTAIR reprograms chromatin state to promote cancer metastasis, Nature, 2010, 464, 1071-1076.

45 G. Rizki and L. A. Boyer, Long noncoding epigenetic control of transcription to cardiovascular development and disease, Circ. Res., 2015, 117, 192-206. 